\title{
Entre "solos" e "terras": Etnopedologia, assentamentos rurais e processos participativos
}

\section{Between "soils" and "lands": etnopedology, rural settlements and participatory processes.}

\author{
Alberto Bracagioli Neto ${ }^{(1)}$ \\ Flávia Charão Marques ${ }^{(2)}$ \\ Paulo César do Nascimento (3) \\ Ingrid Bergman Inchausti de Barros ${ }^{(4)}$ \\ Gustavo Vodzik(5) \\ Nickolas Menezes ${ }^{(6)}$ \\ Patrícia Lima de Lima(7)
}

\begin{abstract}
Resumo
A atividade de produção agrícola tem presença pequena, mas de importância expressiva na região Metropolitana de Porto Alegre (RMPA). Esta importância tem motivado a busca por formas de consolidação desta atividade, principalmente a agricultura familiar. Com o objetivo de avaliar as características dos solos, relacionando-os as percepções e avaliações dos agricultores locais, dois assentamentos da RMPA foram visitados, e lotes de moradia e produção familiar escolhidos para este trabalho. Foram utilizados recursos de entrevista semiestruturada, caminhamento e escolha de algumas glebas para amostragem e observação dos solos. Os resultados mostraram que estes solos têm limitações ao uso, como textura arenosa, baixa fertilidade natural e drenagem restrita em algumas áreas. Os assentados conseguem distinguir estas características, e para isto foi importante a comparação com os solos de suas localidades de origem. Este público demonstrou maior familiaridade com abordagens no campo, evidenciando serem estas as formas mais produtivas de diálogo com técnicos e pesquisadores. Foi percebida a identidade entre várias observações do público, e critérios e atributos técnico-científicos utilizados em levantamentos e avalição de aptidão de uso das terras, mostrando a aproximação entre estas diferentes formas de saber, e viabilizando trabalhos no sentido de planejamento de sistemas de produção sustentáveis, de forma participativa
\end{abstract}

Palavras-chave: agroecologia; etnopedologia; diagnostico participativo.

\footnotetext{
${ }^{1}$ Universidade Federal do Rio Grande do Sul, Porto Alegre, RS, Brasil abracagioli@gmail.com;

${ }_{2}$ Universidade Federal do Rio Grande do Sul, Porto Alegre, RS, Brasil flavia.marques@ufrgs.br;

${ }^{3}$ Universidade Federal do Rio Grande so Sul, Porto Alegre, RS, Brasil pcnasc@ufrgs.br;

${ }^{4}$ Universidade Federal do Rio Grande do Sul, Porto Alegre, RS, Brasil ingridbarros7@hotmail.com;

${ }^{5}$ Universidade Federal do Rio Grande do Sul, Porto Alegre, RS, Brasil gustavovodzik@gmail.com;

${ }^{6}$ Universidade Federal do Rio Grande do Sul, Porto Alegre, RS, Brasil ckmenezes_ufrgs@yahoo.com.br;

${ }^{7}$ Universidade Federal do Rio Grande do Sul, Porto Alegre, RS, Brasil patricialima.agro@yahoo.com.br Artigo recebido em: 29/06/2018. Aceito para publicação em: 04/02/2019.
} 


\begin{abstract}
Although its small presence, agricultural activities have significant importance in in Porto Alegre metropolitan region (PAMR), Rio Grande do Sul State. This fact has reflected in efforts to consolidation of this activities, especially in family farms model. With the aim to evaluate soil characteristics, as well as meet the perceptions and evaluations of producers, two settlements in PARM were visited, and areas of housing and production of family farms were choose for this work. There were used tools like semi-structured interviews, walking, and some plots were selected for soils sampling and observations. These results showed that settlements soils have limitations for agricultural use, like low natural chemical fertility, sandy texture and restrictions to drainage. Local producers can distinguish these characteristics, and for this it was important to make some comparisons with land of their origin localities. Local people showed easier comprehension of field approaches, highlighting the need of these tools for dialogue between technicians, researchers and target people. It was possible to recognize identities between local people observations and technical-scientific attributes used in land suitability evaluations, showing rapprochement between these types of knowledge, allowing planning of sustainable productions systems, in participatory way.
\end{abstract}

Keywords: agroecology; etnopedology, participatory appraisal.

\title{
Introdução
}

A região metropolitana de Porto Alegre (RMPA) teve o início de sua formação ligada a atividades agrícolas. A colonização da região teve predominância de estâncias de gado e produção de charque para o comércio, sendo que no início do século XX começam a se destacar áreas de produção capitalista de arroz (DUVOISIN, 2008). Atualmente a RMPA é a área mais densamente povoada do Rio Grande do Sul, concentrando 37,7\% da população total do Estado, mais de 4 milhões de habitantes (SEPLAN, 2016). Estes dados indicam um processo crescente de concentração de população, com implicações em aspectos de infraestrutura e reflexos na qualidade de vida. Por outro lado, possui diversos assentamentos de reforma agrária, de acordo com o Censo Agropecuário de 2006 (IBGE, 2010), Porto Alegre tem 294 estabelecimentos agropecuários de produtores individuais, ocupando cerca de 5600 ha. Percebe-se, assim, a presença de vazios urbanos significativos, e espaços caracterizados como meio rural (IBGE, 2010; GRANDO \& MIGUEL, 2002). Embora com uma série de descontinuidades, tem havido ações na direção da superação de problemas gerados pela crescente urbanização. Neste 
sentido, reconhece-se no redirecionamento da atividade agrícola parte destes esforços, em especial, tomada a potencialidade da agricultura familiar no que tange à inclusão social e produtiva. À agricultura familiar tem sido reputada não só a capacidade de adaptação a diferentes contextos, mas também a significativa contribuição à produção de alimentos, o que impacta positivamente a segurança alimentar e nutricional das comunidades rurais e urbanas (MDA, 2009; SCHNEIDER, 2003).

A formação dos assentamentos, dentro do processo de Reforma Agrária em âmbito nacional, visa promover a democratização da estrutura fundiária, a produção de alimentos básicos e a geração de trabalho e renda, entre outros (INCRA, 2011). Outro aspecto a considerar é que muitos estudos constatam mudanças positivas na dinâmica socioeconômica de regiões ou municípios que passam a contar com assentamentos rurais e, em parte, também nas condições socioeconômicos e de qualidade de vida dos assentados. (ALVES \& SILVEIRA, 2008). Segundo a COPTEC (2011), entre os 34 municípios que compõem a RMPA, existem Assentamentos rurais (Federais ou Estaduais) em seis municípios, envolvendo cerca de 1100 famílias (Quadro 1).

No entanto, apenas assentar as famílias não garante sua permanência e, tampouco, a contribuição para a região, sendo necessário que se estabeleçam ações de diagnóstico e planejamento para consolidação destas iniciativas. Neste sentido, muitas têm sido as críticas aos processos verticais e exógenos de planejamento, fazendo surgir variadas abordagens que sugerem a participação das comunidades envolvidas em tais ações (VERDEJO, 2006; GOMES \& VILELA, 2004). A necessidade de se buscar sistemas de produção adaptados à realidade local, no que diz respeito às condições e potencialidades ambientais, econômicas, sociais e culturais, tem levado ao desenvolvimento de abordagens diferenciadas no diagnóstico e avaliação do uso dos recursos naturais.

Este trabalho teve como objetivos: i) avaliar, de forma participativa, aspectos sobre os recursos naturais, com ênfase em solos, em assentamentos 
rurais da RMPA; ii) conhecer as experiências, percepções, avaliações e critérios para o uso e manejo da terra por parte dos assentados nas localidades de trabalho, como forma de aumentar a compreensão sobre o lógica e organização das atividades implementadas pelos agricultores familiares.

Quadro 1. Assentamentos rurais de reforma agrária da Região Metropolitana de Porto Alegre

\begin{tabular}{|c|c|c|}
\hline Municípios & Assentamentos & Número de famílias \\
\hline Capela de Santana & São José & 15 \\
\hline \multirow{7}{*}{ Eldorado do Sul } & Faz. S. Pedro & 103 \\
\hline & Apolônio de Carvalho & 72 \\
\hline & Colônia Nonoaiense & 13 \\
\hline & Integração Gaúcha & 74 \\
\hline & Padre Josimo & 24 \\
\hline & Belo Monte & 95 \\
\hline & Lanceiros Negros & 7 \\
\hline Guaíba & Dezenove de Setembro & 36 \\
\hline Montenegro & Vinte e Dois de Novembro & 20 \\
\hline \multirow{4}{*}{ Nova Santa Rita } & Itapui/Meridional & 68 \\
\hline & Capela & 101 \\
\hline & Sino & 13 \\
\hline & Santa Rita de Cassia II & 101 \\
\hline Viamão & Viamão-Filhos de Sepé & 375 \\
\hline TOTAL & & 1117 \\
\hline
\end{tabular}

Fonte: adaptado de COPTEC (2011)

\section{Metodologia}

\section{Características do meio físico}

A região abrangida pelos municípios da RMPA está circunscrita às coordenadas geográficas 29 20' S e 30 40' S, e 50 20' e 52 10'W. Encontra-se 
dentro do bioma Pampa, sendo composta por duas topografias distintas: os terrenos mais baixos, suavemente ondulados e as encostas do escudo Cristalino (HASENACK, 2008; CPRM, 2008). É inserida em transições entre as principais regiões fisiográficas do Rio Grande do Sul, como a Serra do Sudeste e a Depressão Central do Estado (sudoeste); Depressão Central em transição com o início da Encosta da Serra do Nordeste (norte), e à Planície Litorânea, a leste. A classificação climática destas regiões, pelo Sistema de Koppen é "Cfa" (clima subtropical úmido sem estiagem), e precipitação anual entre 1100 e $1700 \mathrm{~mm}$. Os solos representativos são, respectivamente, as classes dos Planossolos, Gleissolos e Organossolos, nas áreas de várzea, e Argissolos Vermelho-Amarelos, Cambissolos Háplicos e Neossolos Litólicos, nas áreas de relevo suave ondulado e ondulado na Depressão Central e Escudo Sul-riograndense (STRECK et al., 2008). De forma geral, existem limitações quanto ao uso do solo, devido à fertilidade natural baixa e também problemas de erosão, drenagem e alagamentos em diversas áreas. Nos campos baixos se caracterizam as várzeas que são áreas que se alagam em determinados períodos do ano, tornando solos aptos para o cultivo de arroz irrigado.

Características dos modelos de estudos

Este tabalho foi realizado de forma a valorizar o protagonismo dos agricultores e seus familiares, relacionando-se a abordagem técnico-científica às observações, percepções e avaliações feitas pelos agricultores, e originadas a partir da vivência e do trabalho diários dos mesmos na terra. Assim, procurou-se lançar as bases para uma abordagem etnopedológica (ALVES et al., 2005; ARAÚJO et al. 2013).

Contatos foram estabelecidos com lideranças de movimentos sociais, e também com técnicos da Cooperativa de Prestação de Serviços técnicos 
(COPTEC), que atua em assentamentos. Foram definidas as ações nos assentamentos "Santa Rita de Cássia II" (SRC), no município de Nova Santa Rita, e "São Pedro" (SPE), no município de Eldorado do Sul.

O assentamento São Pedro, constituído em 1986, tem área total com cerca de 2250 ha, sendo assentadas 100 famílias (COPTEC, 2011). Neste assentamento, o trabalho foi feito mais especificamente com um grupo de produção orgânica (base agroecológica), com presença de 7 famílias, pertencentes aos diferentes blocos do assentamento. A produção era basicamente de olericultura e fruticultura, procurando-se seguir um manejo de base agroecológica. Pode-se notar, no entanto, que esta modalidade de produção encontrava-se em diferentes graus de consolidação, caracterizando, de forma geral, um sistema de transição ou implantação (CAMARGO, 2007).

O assentamento Santa Rita de Cássia II tem extensão total de 1660 ha, aproximadamente, sendo assentadas 103 famílias (INCRA, 2009). As áreas de várzea são ocupadas por produção de arroz, em sistemas de grupo de produção, reunindo oito a dez famílias por grupo. As áreas “altas”, em solos bem drenados, são utilizadas principalmente com olericultura. Muitos assentados tem uma parte de sua área em cada região, sendo as terras altas reservadas para a moradia, mas a maior extensão nas terras baixas. Outras famílias têm lotes "inteiros", normalmente na posição de transição entre as regiões altas e baixas. Nesse assentamento, trabalhou-se com dez famílias ou lotes, predominando a produção de olerícolas, em sistemas de produção agroecológico. Em ambos assentamentos, características como a região de origem e a opção por determinados sistemas de produção ajudaram a definir grupos dentro do conjunto de famílias assentadas (MIRANDA, 2007).

\section{Metodologia}

Reuniões iniciais foram estabelecidas nos assentamentos, com o objetivo de divulgar e sensibilizar os assentados para a participação no 
trabalho, bem como realizar a coleta de impressões sobre o tema proposto (diagnóstico sobre os recursos naturais e influência destes na vida e trabalho das famílias). O perfil dos participantes destas reuniões, que acabariam constituindo o "público-alvo" em cada assentamento, mostrou certa homogeneidade, no que diz respeito a histórico, atividades atuais e perspectivas, podendo-se definir as reuniões como de um "grupo focal" (MINAYO, 2010).

Os relatos feitos pelos participantes foram reforçados, a partir do uso de diagramas e desenhos por eles formulados. Nesses, os representantes de cada setor geográfico representavam aspectos principais, como a localização de escolas e outros equipamentos sociais, localização dos lotes, e distribuição espacial das características e uso das terras, caracterizando o método de mapeamento participativo (CHAMBERS, 1992). Pode-se sentir, já no início, interesse e disposição para a participação no projeto.

Em um segundo momento, o trabalho foi desenvolvido nos lotes dos assentados. Nestes dias, após um rápido encontro dos assentados, técnicos da COPTEC e professores e estudantes da universidade, o trabalho era feito em cada lote. Nesta etapa, procurou-se fazer incialmente uma breve entrevista semiestruturada (VERDEJO, 2006; MINAYO, 2010), com algumas questões balizadoras, mas permitindo às famílias ressaltar aspectos mais importantes, para elas próprias, sobre sua origem, trajetória (nos seus lugares de origem e a partir da entrada no assentamento), dificuldades encontradas, bem como aspectos ligados ao trabalho e produção, como o uso da terra e organização do trabalho. Após este momento, foi feita uma caminhada pelo lote, observandose as áreas de maior interesse por parte do agricultor, no que diz respeito a diagnóstico sobre características e qualidade do solo, em um procedimento semelhante ao definido como "caminhada em transecto" (CHAMBERS, 1992). Nestas glebas (em geral duas a quatro por lote), foi feita a observação sobre as características do solo, por meio de descrição morfológica expedita do perfil representativo (SANTOS et al., 2005), procurando enquadra-lo em uma classe 
definida por sistemas taxonômicos (EMBRAPA, 2013), bem como avaliar, de forma participativa, alguns aspectos sobre limitações, problemas, bem como práticas de manejo para superação e/ou convivência com os mesmos (RESENDE et al., 2002). Ao mesmo tempo, foram coletadas amostras de solo, na camada de 0 a $20 \mathrm{~cm}$, para análise de principais atributos físicos e químicos.

A atividade feita nos lotes contava com a participação da equipe executora do projeto, de representantes da família assentada no lote, de outros assentados pertencentes ao grupo trabalhado, e, em alguns casos, de técnicos ligados à COPTEC. Este foi um fator de enriquecimento do diálogo, da troca de ideias e da aproximação entre saberes locais e técnico-científicos. Computando-se os dois assentamentos trabalhados, chegou-se a 17 lotes e 38 glebas trabalhados, com coletas de amostras de solo, e observações sobre os perfis do solo em 16 pontos. A olericultura foi a principal atividade nas glebas amostradas (21 glebas), também destacando-se as atividades de lavouras, campo ou pastagem e pomar de frutíferas (em torno de cinco glebas cada)

Por último, foi traçado um cronograma com as etapas seguintes do trabalho, com ênfase no retorno da equipe executora ao assentamento com os resultados das análises realizadas, de forma a avaliar estes dados.

\section{Resultados e Discussão}

Uso da terra e características dos solos

Os resultados das análises realizadas indicaram, do ponto de vista químico, a dependência do histórico de uso e manejo das áreas. $\mathrm{O}$ uso em olericultura (hortas), e o sistema de produção utilizado, tiveram reflexos em atributos como $\mathrm{pH}$, teores de matéria orgânica e fósforo (Tabela 1). Observamse os maiores valores de $\mathrm{pH}$ e teores de fósforo em glebas sob uso com hortas 
(olericultura), pelas grandes adições de adubos orgânicos, a cada dois ou três ciclos de produção. Por outro lado, os teores de matéria orgânica permanecem baixos, pela facilidade de decomposição deste material, e também pela textura arenosa dos solos (GEBRIM et al., 2010; REIS et al., 2014).

Tabela 1: Distribuição do número de glebas por classes de referência, segundo CQFS (2004), para atributos químicos do solo.

\begin{tabular}{lccccccccc}
\hline & \multicolumn{3}{c}{$\mathrm{pH}$} & \multicolumn{3}{c}{ MO } & & $\mathrm{P}$ \\
\hline $\begin{array}{l}\text { Uso da } \\
\text { terra }\end{array}$ & $\begin{array}{l}\text { Al. a } \\
\text { Mal. }\end{array}$ & Médio & $\begin{array}{l}\text { Bx a } \\
\text { Mbx }\end{array}$ & $\begin{array}{l}\text { Al. a } \\
\text { Mal. }\end{array}$ & Medio & $\begin{array}{l}\text { Bx a } \\
\text { Mbx }\end{array}$ & $\begin{array}{l}\text { Al. a } \\
\text { Mal. }\end{array}$ & Medio & $\begin{array}{l}\text { Bx a } \\
\text { Mbx }\end{array}$ \\
\hline Horta & 15 & 2 & 4 & 0 & 0 & 21 & 19 & 0 & 2 \\
Outros* & 3 & 6 & 5 & 0 & 2 & 12 & 4 & 2 & 8
\end{tabular}

Al: alto; Mal; muito alto; Md: médio; Bx: baixo; Mbx: muito baixo. MO: teor de matéria orgânica; P: teor de fósforo

* pastagem, frutíferas, lavouras.

Org: do Autor, 2018

As características dos solos presentes nos assentamentos estudados derivam principalmente da litologia e do relevo. Destacam-se a ocorrência de solos profundos, tendência de mudança textural ao longo do perfil do solo, em geral a partir de profundidade de 30 a $50 \mathrm{~cm}$., a coloração que varia de vermelho a acinzentado, de acordo com a drenagem natural do solo, esta por sua vez condicionada à posição do relevo (KAMPF \& CURI, 2012).

Estes aspectos, ligados diretamente à capacidade de uso e suscetibilidade a degradação, foram de imediato percebidos pelos assentados, no momento da chegada a estes locais. Para isto, teve grande influência a origem dos mesmos, vindos em sua maioria do Planalto ao norte do Estado, onde a geologia formada a partir de material basáltico se reflete em solos de textura argilosa, e altos teores de óxidos de ferro, influenciando propriedades como agregação do solo e potencial nutricional para as plantas cultivadas. 
Identidade e estranhamentos: as percepções e saberes locais.

A preocupação com a qualidade do solo e dos recursos naturais, e mesmo com a qualidade de vida, está expressa na opção por sistemas de produção de base agroecológica. Observa-se, porém, uma variação entre os produtores em relação ao nível alcançado dentro de processos de transição, sendo que a maioria se situa em um estágio de substituição de insumos (GLIESSMANN, 2009).

$\mathrm{Na}$ primeira reunião no SPE, alguns relatos mostraram a dificuldade de adaptação a esta nova condição, em relação ao recurso natural solo: "solo arenoso, principalmente nas áreas baixas, diferentes da nossa região, que era de terra fértil". A tentativa de reproduzir, no assentamento, os sistemas de produção do local de origem, também se constituiu em um entrave. Um outro assentado, vindo da região Norte, sintetiza o pensamento original dos assentados, ao planejar o uso da terra com grandes lavouras: "barriga de pobre, cabeça de rico". A granulometria do solo (teores de argila, silte e areia) tem sido sistematicamente incluída, pelos agricultores, como um atributo dos mais perceptíveis, tendo ainda um caráter primordial na definição do potencial de uso da terra e dos sistemas de preparo e condução das culturas. Isto é mais ressaltado quando a experiência do agricultor inclui o trabalho em áreas com diferentes texturas, permitindo observar as diferenças resultantes nas propriedades do solo (FINATO et al, 2015).

Observações semelhantes foram detectadas no SRC. Na primeira reunião geral, foi relatado, pelos agricultores, alguns aspectos sobre a textura e a morfologia do solo em geral, associando isto à qualidade original do solo: "A terra é fraca, tem poucos centímetros de areia e depois vira uma tabatinga". A expressão "tabatinga", neste caso, caracteriza o aumento de argila em profundidade, com consistência do solo mais dura, característico de solos de várzea da região, os Planossolos (EMBRAPA, 2013). A percepção de características do solo levou um assentado a citar uma classe estabelecida 
pelo sistema técnico-científico, comparando as terras do assentamento e de seu local de origem, o "Alto Uruguai": "lá tinha as terras de pousio, os Latossolos. Aqui é mais diversificado, tem Latossolos, mas também tem terra arenosa, mais areia do que terra. Isto é problema por causa da erosão”. É interessante notar que, nesse caso, é feita uma observação com base em características morfológicas do solo (homogeneidade do solo em suas diferentes secções, coloração avermelhada, grande profundidade), que normalmente caracterizam a classe taxonômica dos Latossolos (EMBRAPA, 2013; Streck et al., 2008). A região onde se insere o SRC não apresenta esta classe de solo, porém as semelhanças dos solos de algumas áreas deste assentamento com os Latossolos do Alto Uruguai, levaram o agricultor a fazer esta relação, que apresenta lógica dos pontos de vista técnico e cognitivo.

A princípio, pode-se perceber que a textura arenosa é mais atribuída aos solos de várzea, porém a experiência do trabalho diário em áreas de "coxilhas" (relevo suave ondulado) também levou a observações mais apuradas sobre esta característica: “As pessoas pensam que é diferente de lá de baixo, mas também tem muita areia. Só é mais seco.”. Esta observação mostra outro atributo bastante importante. Apesar de não ter sido tão citada, a cor, nesse caso, é uma característica associada por uma assentada, na diferenciação entre tipos de solos e ambientes no seu lote: "aqui em cima tem terra mais vermelha".

Alguns outros atributos, tanto do solo como do ambiente, também são ressaltados nas observações dos assentados. Como o assentamento conta com uma grande área sob drenagem restrita, alguns fenômenos sobre a dinâmica de elementos são percebidos: "Às vezes se se vê uma ferrugem amarela, que parece ser resíduo de agrotóxico. A adubação é em parte perdida, a água puxa a gordura para baixo”. Em áreas de relevo plano ou suave ondulado, com drenagem com alguma restrição, é feita referência a ocorrência de concreções na forma de "chumbinhos" ou "pedrinhas". Em lote do SPE, este material se apresenta em volumes maiores, de cerca de até $30 \mathrm{~cm}$ de diâmetro: "tem 
pedras espalhadas pela gleba”. Apesar de não ter sido feita uma análise específica para caracterização e definição de origem destas feições, é bem plausível que sejam originadas de concreções de ferro, por processos pedogenéticos de redução, oxidação e precipitação acumulativa na forma de óxidos de ferro (KAMPF \& CURI, 2012; MIGUEL et al., 2013).

É perceptível a preocupação dos assentados com degradação do solo e dos recursos naturais, como consequência de um histórico de uso intensivo da terra, em sistema de produção convencional, antes da formação do assentamento. As observações e a experiência dos agricultores, associados à assistência técnica, levam a algumas escolhas no preparo e manejo do solo, bem como condução das culturas. Por exemplo, a opção por uso de cobertura do solo é explicada por uma assentado: "a palha desmancha e dá gordura na terra; se não colocar palha, vai ter que capinar 3 ou 4 vezes mais”.

Desta forma, foi possível perceber que o conjunto de observações, percepções e conhecimentos dos assentados, em relação aos recursos naturais disponíveis e seu uso mais adequado, integra a experiência adquirida em um longo do tempo de trabalho e convivência com a terra, sua sistematização e interpretação, e as medidas e práticas escolhidas para seu manejo, de forma a estabelecer formas sustentáveis de produção. Destaca-se a capacidade de compreender estas características de forma multidimensional, integrando aspectos técnicos, econômicos, sociais e culturais, o que fica implícito nos critérios utilizados para a definição do uso e manejo do solo. Barrera Bassols \& Zinck (2003) identificam este conjunto de percepções como "corpus", referente aos conhecimentos apropriados pelos agricultores locais por meio do trabalho, observação e formação de conhecimento; e "práxis", consistindo nas opções sobre uso e manejo da terra, e sua execução. 
As relações entre formas do saber: reflexões e projeções

A partir do roteiro traçado junto aos assentados, a etapa final do trabalho consistiu em reuniões de avalição dos resultados sobre as características do solo, e de todo o projeto em si. Neste momento, situações diferentes caracterizaram esta etapa, em cada um dos assentamentos. No SPE, com base nos resultados das análises de solo, foi feita uma reunião para discussão dos resultados obtidos, e também uma avaliação sobre a execução do projeto, em sua totalidade. A preparação desta reunião ocorreu com base na elaboração de material visual, para exposição ao conjunto de assentados participantes, e também técnicos da COPTEC, procurando-se levantar temas para uma participação mais efetiva por parte do público. Foram ainda entregues laudos individuais, onde para cada atributo do solo (teores de argila, $\mathrm{pH}$, teores matéria orgânica e nutrientes, entre outros), procurou-se apresentar os níveis em classificação em níveis de "muito baixo" a "muito alto”, conforme CFQS (2004).

Foi percebida, por parte dos técnicos, uma maior dificuldade em estimular uma postura mais participativa para as análises e a avaliação dos resultados, ao contrário do que acontece em atividades do campo, onde o grupo de assentados demonstra maior segurança nas observações. As menções aos tipos de solo ("solo com pedra, solo colorido, pedrisco acima e terra em baixo") são mais facilmente relacionadas com problemas e limitações, que os resultados analíticos.

Apesar destes problemas, alguns pontos mostraram-se interessantes para permitir reflexões para o grupo: "fazemos muita adubação orgânica, mas a matéria orgânica ainda é baixa.” Os técnicos ligados a produção no assentamento também demonstraram preocupação com aspectos ambientais: "temos que tomar cuidado com os metais pesados, zinco e cobre".

As observações e avaliações feitas nessa reunião serviram para algumas alterações na dinâmica, por ocasião de reunião de avaliação no SRC. 
Procurou-se, já em um primeiro momento, utilizar recursos visuais mais dinâmicos, onde os assentados também pudessem participar, inclusive acrescentando observações ou itens que considerassem pertinentes. Assim, foi utilizado um álbum seriado. A abordagem procurou não priorizar tanto os resultados das análises de solo, mas observações gerais, que podiam ser constatadas pelos assentados. Entre os pontos abordados, mais uma vez foi destacado o caráter da drenagem dos solos: "Vivemos em uma ilha, no inverno o solo sofre pelo excesso de água, no verão fica muito duro e seco". Apesar de alguns problemas e da necessidade de algumas mudanças nos sistema de produção, os assentados reiteraram a satisfação pela adoção de uma maior diversidade na produção, e práticas com base em sistemas de produção agroecológica: "Quando chegamos no assentamento, o monocultivo tinha acabado até com os passarinhos, mas agora eles estão voltando". Foi importante perceber, de qualquer forma, que para que o trabalho realizado possa efetivamente trazer ganhos ao nosso público-alvo, é fundamental a participação do corpo técnico na assessoria dos assentados, bem como a viabilização de formas de dar continuidade ao trabalho. Critérios mais aprofundados de avaliação da qualidade do solo, bem como o planejamento de formas de manejo, dentro dos sistemas de produção adotados, são tópicos que podem ser abordados na continuidade do trabalho realizado, assim como a maior valorização de atividades de campo, um ambiente de maior familiaridade em relação às práticas e as rotinas dos agricultores, e um maior grau de associação entre os resultados analíticos obtidos e as observações diretas feitas conjuntamente, por técnicos e agricultores.

\section{Considerações Finais}

Os solos presentes nos assentamentos têm algumas características que implicam em limitações e necessidades de práticas de conservação, como textura arenosa, variações de granulometria ao longo do perfil e drenagem 
restrita. Os atributos químicos, em superfície, já sofreram alterações importantes de acordo com o histórico e uso e manejo recentes.

As percepções dos assentados sobre as características das terras nos assentamentos, enriquecidas pela sua origem de outras regiões, permitiram comparações que facilitam a caracterização do solo, bem como os critérios para adoção de práticas de uso e manejo.

A maior identificação e o maior "empodeiramento" dos assentados, nas observações e avaliações sobre o solo, ocorreu quando as atividades e discussões foram realizadas diretamente no campo. Isto mostra a necessidade de experiência, de adaptações de abordagens e procedimentos, por parte do grupo executor.

Ocorre uma identidade entre percepções e avaliações dos agricultores locais e atributos e características utilizadas como critérios na análise técnico cientifica, principalmente quanto a atributos físicos e morfológicos, ligados à formação dos solos e a aptidão de uso da terra. Isto reforça a perspectiva de trabalhos, avaliação e planejamento participativo na definição de sistemas de produção.

\section{Agradecimentos}

Aos Ministérios da Educação (MEC) e Desenvolvimento Agrário (MDA), pelo apoio financeiro por meio do Edital PROEXT 2016; aos técnicos da COPTEC, pelo apoio em todas as etapas do projeto; aos assentados, pela oportunidade de com eles conviver e aprender.

\section{Referências}

ALVES; A. G. C. A E MARQUES, J. G. W. Pedologia, uma nova ciência? . In: Tópicos em Ci. Solo, 4:321:344. Soc. Bras. Ci. Solo, Viçosa (MG), 2005.

ALVES, F. D. E SILVEIRA, V. C. P. A metodologia sistêmica na geografia agrária: um estudo sobre a territorialização dos assentamentos rurais. Sociedade e Natureza, 20(1), 125-137, 2008. https://doi.org/10.1590/S1982-45132008000100009 
ARAÚJO, A. L.; ALVES, A. G. C.; ROMERO, R. E. E FERREIRA, T. O. Etnopedologia: uma abordagem das etnociências sobre as relações entre sociedades e solo. Ciência Rural, 5: 854860, 2013. https://doi.org/10.1590/S0103-84782013000500016

BARRERA BASSOLS, N.; ZINCK, J. A.. Ethnopedology: a worldwide view on the soil knowledge of local people. Geoderma, 111:171-195, 2003. https://doi.org/10.1016/S00167061(02)00263-X

CAMARGO, P. Fundamentos da transição agroecológica: racionalidade ecológica e campesinato. Agrária, 7, 156-181, 2007. https://doi.org/10.11606/issn.1808-1150.v0i7p156$\underline{181}$

CHAMBERS, R. The origins and practice of participatory rural apraisal. World Development, 22:953-969, 1994. https://doi.org/10.1016/0305-750X(94)90141-4

CPRM - COMISSÃO DE PESQUISA EM RECURSOS MINERAIS. Mapa geológico do Estado do Rio Grande do Sul. Porto Alegre (RS), 2008.

CQFS - COMISSÃO DE QUÍMICA E FERTILIDADE DO SOLO RS-SC. Manual de Adubação e Calagem para os Estados de SC e RS. Sociedade Brasileira de Ciência do Solo - Núcleo Regional Sul, Porto Alegre, 2004. 404 p.

COPTEC - Cooperativa de Prestação de Serviços Técnicos Ltda. -. Plano de recuperação de Assentamento - Fazenda São Pedro. Min. Des. Agrário, 2011. 162 p.

CORREIA, J. R.; LIMA, A. C. S.; ANJOS, L. H. C. O trabalho de pedólogo e suas relações com as comunidades rurais: observações com agricultores familiares no norte de minas Gerais. Cadernos Ciência e Tecnologia, 21:447-467, 2004.

DUVOISIN, L. A. A. O Sindicato Arrozeiro do Rio Grande do Sul e o Estado: reflexões sobre a institucionalização de interesses e a intervenção econômica na crise da Primeira República. 27/09/2008. Disponível em: http://www.historia.uff.br/polis/files/texto_13.pdf Acesso em: 12 dez. 2016.

EMBRAPA - Empresa Brasileira de Pesquisa Agropecuária. Centro Nacional de Pesquisa de Solos. Sistema Brasileiro de Classificação de Solo. 3.ed. Rio de Janeiro: Embrapa, 2013. 353 p.

GLIESSMAN, S. R. Agroecologia - Processos agroecológicos em agricultura sustentável (quarta edição). Editora UFRGS, Porto Alegre (RS), 2009. 640 p.

GOMES, M. A. O.; VILELA, G. F. Uma dimensão subjetiva da participação. In: Participação na Extensão Rural - Experiências Inovadoras de Desenvolvimento Local. Tomo Editorial. Porto Alegre (RS), 2004. p $227-244$.

GRANDO, M. Z.; MIGUEL, L. A. (org.). Agricultura na região metropolitana de Porto Alegre. Editora UFRGS, Porto Alegre (RS), 2002. $157 \mathrm{p}$.

FINATO, T.; NASCIMENTO, P.C.; BECK, F. L.; TORNQUIST, C. G.; CAETANO, L. A. M.; FEDRIZZI, T. Z. Percepções locais sobre solo e seu uso no município de Gravataí - RS. Rev. Bras. Ci. Solo, 2015. https://doi.org/10.1590/01000683rbcs20140514

GEBRIM, F. O., NOVAES, R. F.; SILVA, I. R. Mobility of organic and inorganic phosphorus under different levels of phosphate and poultry litter fertilization in soils. Rev. Bras. Ciência do solo, v. 34; p. 1195-1205, 2010. https://doi.org/10.1590/\$0100-06832010000400019

GUTIÉRREZ, L. A. L. Agroecologia e desenvolvimento de assentamentos de reforma agrária: ação coletiva e sistemas locais de conhecimento e inovação na região metropolitana de porto alegre. 2012. 407 f. Tese (Doutorado) - Curso de Pós-graduação Interdisciplinar em Ciências Humanas, Universidade Federal de Santa Catarina, Centro de Filosofia e Ciências Humanas. Florianópolis, 2012.

HASENACK, H. (Coord.). Diagnóstico Ambiental de Porto Alegre: Geologia, Solos, Drenagem, Vegetação/Ocupação e Paisagem. Porto Alegre: Secretaria Municipal do Meio Ambiente (SMAM), 2008. 84 p.

IBGE - Instituto Brasileiro de Geografia e Estatística. Censo Demográfico 2010. Disponível em https://cidades.ibge.gov.br/. Acesso em 25 mar. 2016

INCRA - Instituto Nacional de Colonização e Reforma Agrária (INCRA). Reforma Agrária no Brasil. Disponível em www.incra.gov.br/reformaagraria. Acesso em fevereiro de 2011. 
INCRA- Instituto Nacional de Colonização e Reforma Agrária. Relatório Ambiental do Projeto de Assentamento Santa Rita de Cássia II. Min. Des. Agrário, Porto Alegre, 2009. 110 p.

KAMPF, N.; CURI, N. Formação e evolução do solo (Pedogênese). In: Ker, J. C. et al (editores). Pedologia (Fundamentos). Sociedade Brasileira de Ciência do Solo. Viçosa (MG), 2012. P. 207 301.

MIGUEL, P.; DALMOLIN, R. S. D.; PEDRON, F. A.; FINK, J. R. E MOURA-BUENO, J.M. Caracterização de plintitas e petroplintitas em solos da Depressão Central do Rio Grande do Sul. Ciência Rural, 999-1005, 2013. https://doi.org/10.1590/S0103-84782013005000065

MDA - Ministério do Desenvolvimento Agrário. Agricultura familiar no Brasil e o Censo Agropecuário 2006. Brasília (DF), 2009. 14 p.

MINAYO, M. C. S. Trabalho de campo: contexto de observação, interação e descoberta. In: Pesquisa social: teoria, método e criatividade. Minayo, M. C. S.; Deslandes, S. F. e Gomes, R. Editora Vozes, Petrópolis (RJ), 2010. p 61-78.

MIRANDA, R.S. Arranjos institucionais e o acesso e uso de recursos naturais em assentamentos rurais do semiárido paraibano. Sociedade e Natureza, 29:39-52, 2017. https://doi.org/10.14393/SN-v29n2-2017-6

REIS, C. E. S.; DICK, D. P.; CALDAS, J. S.; BAYER, C. Carbon sequestration in clay and silt fractions of Brazilian soils under conventional and no-tillage systems. Scient. Agrícola, 4:292-301, 2014. https://doi.org/10.1590/0103-9016-2013-0234

RESENDE, M; LANI, J.L. E CURI, N. Reflexões sobre o uso do solos brasileiros. In: Tópicos em Ciência do Solo, volume II. Soc. Bras. Ci. Solo, Viçosa, 2002. p. 593 - 644 .

SANTOS, R. D.; LEMOS, R. C.; SANTOS, H. G.; KER, J. C.; ANJOS, L. H. C. Manual de descrição e coleta de solos no campo. Sociedade Brasileira de Ciência do Solo - Centro Nacional de Pesquisa de Solos - EMBRAPA. Rio de Janeiro (RJ), 2005. 92 p.

SCHNEIDER, S. teoria social, agricultura familair e pluriatividade. Rev. Bras. de Ciências Sociais, 18:99-121, 2003. https://doi.org/10.1590/S0102-69092003000100008

SEPLAN - ATLAS SOCIOECONÔMICO RIO GRANDE DO SUL (Org.). Região Metropolitana de Porto Alegre - RMPA. Porto Alegre: Secretaria Estadual de Planejamento, Governança e Gestão. Disponível em http://www.atlassocioeconomico.rs.gov.br/regiaometropolitana-de-porto-alegre-rmpa Acesso em: 25 mar. 2016.

SILVA NETTO, L. F.; INDA, A. V.; NASCIMENTO, P. C.; GIASSON, E. SCHMITT, C.; CURI, N. Characterization and classification of floodplain soils in the Porto Alegre metropolitan region, RS, Brazil. Sci. Agrot., 5: 423-434, 2015. https://doi.org/10.1590/S1413$\underline{70542015000500001}$

STRECK, E. V., KAMPF, N., DALMOLIN, R. S. D., KLAMT, E. V., NASCIMENTO, P. C., SCHNEIDER, P. Solos do Rio Grande Do Sul. Empresa de Assitência Técnica e Extensão Rural (EMATER) - Universidade Federal do Rio Grande do Sul.Porto Alegre, 2008.

VERDEJO, M. E. Diagnóstico rural participativo - Guia prático. Ministério do Desenvolvimento Agrário (MDA), Brasília (DF), 2006. 62 p. 